\title{
Quaternionic-Kähler geometry and almost Kähler $\mathcal{A}$-manifolds
}

\author{
by WŁODZIMIERZ JELONEK (Kraków)
}

\begin{abstract}
The aim of this paper is to give an easy explicit description of 3-K-contact structures on certain $\mathrm{SO}(3)$-principal fibre bundles over quaternionic-Kähler manifolds.

0. Introduction. It has been known since $1975[\mathrm{~K}]$ that any quaternionic-Kähler manifold $\left(M, g_{0}\right)$ of positive scalar curvature admits a natural $\mathrm{SO}(3)$-principal fibre bundle $p: P \rightarrow M$ such that $(P, g)$ is a 3-Sasakian manifold and $p$ is a Riemannian submersion. However for a long time the analogous construction for quaternionic-Kähler manifolds of negative scalar curvature was not given. Recently S. Tanno [T] proved that a natural SO(3)principal bundle also in the case of negative scalar curvature admits a structure similar to 3-Sasakian structure, called by him the nS-structure. Alexandrov, Grantcharov and Ivanov [A-G-I] study the almost complex structures on the Salamon twistor bundle over quaternionic-Kähler manifolds. In particular they prove by generalizing results of Davidov and Muskarov [D-M] that in the case of negative scalar curvature the twistor bundle $(Z(M), g)$ admits an almost Kähler non-Kähler structure $J$ and the Ricci tensor of $Z(M)$ is Hermitian with respect to $J$. They also prove that $(Z(M), g, J)$ satisfies the symmetry condition $G_{2}$ of A. Gray.

In this paper we prove the existence of a quasi 3-K-contact structure on the canonical SO(3)-principal bundle over a quaternionic-Kähler manifold. We use a method different from that of S. Tanno (who gave a more detailed description of this structure). We construct explicitly a homomorphism $F: Q \rightarrow P^{-}$of principal fibre bundles where $Q$ is the natural $\operatorname{Sp}(n) \operatorname{Sp}(1)$ subbundle of the bundle $\mathrm{SO}(M)$ of oriented orthonormal frames of $M$.
\end{abstract}

2000 Mathematics Subject Classification: 53C05, 53C20, 53C25.

Key words and phrases: quaternionic-Kähler manifold, contact structure, principal fibre bundle.

The work was supported by KBN grant 2 P0 3A 01615. 
The class of $\mathcal{A}$-manifolds (for definition see [G]) is an important class of Riemannian manifolds which has appeared in a natural way during the investigation of spaces with volume preserving local geodesic symmetries (see $[\mathrm{B}],[\mathrm{D}-\mathrm{N}]$ ). In our paper $[\mathrm{J}-1]$ we have explicitly constructed a family of locally non-homogeneous $\mathcal{A}$-manifolds parameterized by a real number $c>0$ such that each of these manifolds has a Ricci tensor with two constant eigenvalues. These manifolds are SO(3)-bundles over self-dual Einstein manifolds. In the present paper we consider a related problem and give examples of $\mathcal{A}$-manifolds with two constant eigenvalues on $\mathrm{SO}(3)$-bundles over quaternionic-Kähler manifolds $(M, g)$ generalizing our results from [J-1], [J$3]$. We also prove that the standard Riemannian structure $\left(Z(M), g_{*}\right)$ on the (generalized Salamon $[\mathrm{S}]$ ) twistor bundle $Z(M)$ of a quaternionic-Kähler manifold $M$ is a proper $\mathcal{A}$-structure and that $\left(Z(M), g_{*}\right)$ admits an almost Hodge structure with Hermitian Ricci tensor.

1. Preliminaries. We start with recalling some basic facts concerning the quaternionic-Kähler geometry (see $[\mathrm{S}],[\mathrm{Sw}],[\mathrm{B}]$ ). We denote by $\mathfrak{X}(M)$ the Lie algebra of all local vector fields on $M$. If $D$ is a vector bundle over $M$ then $\Gamma(D)$ denotes the set of all local sections of $D$. We also write $\mathcal{A}^{k}(M)=\Gamma\left(\bigwedge^{k} T M^{*}\right)$.

Let $(M, g)$ be an oriented Riemannian manifold, $\operatorname{dim} M=4 n, n>1$, and $P=P(M, \mathrm{SO}(4 n))$ be the principal fibre bundle of oriented orthonormal frames $u: \mathbb{R}^{4 n} \rightarrow T M$. We denote by $\nabla$ the Levi-Civita connection of $(M, g)$. Let $\mathcal{G} \subset \operatorname{End}(T M)$ be a 3 -dimensional subbundle locally generated by three almost complex structures $\{I, J, K\}$ compatible with the metric $g$ and satisfying the additional condition $I \circ J=-J \circ I=K$. We shall assume that the subbundle $\mathcal{G}$ is parallel with respect to $\nabla$, i.e. $\nabla_{X} A \in \mathcal{G}$ for all $A \in \Gamma(\mathcal{G})$ and $X \in \mathfrak{X}(M)$. A Riemannian manifold with a bundle $\mathcal{G}$ satisfying the above conditions is called a quaternionic-Kähler manifold. Every quaternionic-Kähler manifold is an Einstein manifold. If we regard the curvature tensor of $(M, g)$ as a self-adjoint endomorphism of $\bigwedge^{2} T^{*} M$ then

$$
R_{\mid \mathcal{G}}=\alpha \operatorname{id}_{\mid \mathcal{G}}
$$

where $\alpha$ is a positive multiple of the scalar curvature $\tau$ of $(M, g)$ (see $[\mathrm{Sw}]$, $[\mathrm{G}-\mathrm{L}])$. Note that $\alpha=\tau /(4(n+2))$ (see $[\mathrm{K}]$ and $[\mathrm{G}-\mathrm{L}])$.

The (sphere) subbundle $Z(M)=\left\{A \in \mathcal{G}:\|A\|^{2}=2 n\right\}$ of $\mathcal{G}$ is called the twistor bundle of the quaternionic-Kähler manifold $M$. It will be useful to consider $Z(M)$ as a sphere subbundle of $\bigwedge^{2} T M$ (we identify the bundles $\bigwedge^{2} T^{*} M$ and $\bigwedge^{2} T M$ by means of $\left.g\right)$. With every $\omega \in Z(M) \subset \bigwedge^{2} T M$ we associate an almost complex structure $J_{\omega}$ on $T_{p(\omega)} M$ by the formula

$$
g\left(X, J_{\omega} Y\right)=g(X \wedge Y, \omega) .
$$


Let $\{1, i, j, k\}$ be the standard basis of the quaternion field $\mathbb{H}$. Let $V=$ $\mathbb{H}^{n}$ and $\left\{I_{0}, J_{0}, K_{0}\right\}$ be the complex structures on $V$ defined by $I_{0} x=$ $-x i=\left(-x_{1} i, \ldots,-x_{n} i\right), J_{0} x=-x j=\left(-x_{1} j, \ldots,-x_{n} j\right), K_{0} x=-x k=$ $\left(-x_{1} k, \ldots,-x_{n} k\right)$ where $x=\left(x_{1}, \ldots, x_{n}\right) \in \mathbb{H}^{n}$. On $V$ we have the standard scalar product $\langle x, y\rangle=\sum x_{i} \bar{y}_{i}$. The group $\operatorname{Sp}(n)$ is $\operatorname{Sp}(n)=\left\{A \in \operatorname{End}\left(\mathbb{H}^{n}\right)\right.$ : $\|A x\|=\|x\|$ for $x \in V\}$. The group $\operatorname{Sp}(n) \operatorname{Sp}(1)=\operatorname{Sp}(n) \times_{\mathbb{Z}_{2}} \operatorname{Sp}(1)$ acts on $V$ by the formula $(A, q) x=A x q^{-1}$.

We shall identify the space $\bigwedge^{2} \mathbb{R}^{4 n}$ with $\mathfrak{s o}(4 n)$. The scalar product on $\mathfrak{s o}(4 n)$ is given by $\langle A, B\rangle=-\frac{1}{2} \operatorname{tr} A \circ B$. Note that $\mathfrak{s p}(n) \oplus \mathfrak{s p}(1) \subset \mathfrak{s o}(4 n)$. Let $\pi: \mathfrak{s o}(4 n) \rightarrow \mathfrak{s p}(1)$ be the orthogonal projection with respect to this product. The components of a vector $X \in \mathfrak{g}$ with respect to the decomposition $\mathfrak{g}=$ $\mathfrak{s p}(n) \oplus \mathfrak{s p}(1)$ are denoted by $X^{+}, X^{-}$respectively, i.e. $X=X^{+}+X^{-}$where $X^{+} \in \mathfrak{s p}(n)$ and $X^{-} \in \mathfrak{s p}(1)$. Note that $X^{-}=\pi(X)$ if $X \in \mathfrak{g}$. The algebra $\mathfrak{s p}(1)$ is spanned by $\left\{I_{0}, J_{0}, K_{0}\right\}$. We have $\left\|I_{0}\right\|^{2}=\left\|J_{0}\right\|=\left\|K_{0}\right\|^{2}=2 n$.

Let us define the 1 -forms $\phi_{I_{0}}, \phi_{J_{0}}, \phi_{K_{0}}$ on $\mathfrak{s o}(4 n)=\bigwedge^{2} \mathbb{R}^{4 n}$ as follows: $\phi_{I}(X)=\langle I, X\rangle$ where $I \in\left\{I_{0}, J_{0}, K_{0}\right\}$. Note that for simple bivectors $X \wedge Y$ we have

$$
\phi_{I}(X \wedge Y)=\langle I, X \wedge Y\rangle=g(I X, Y)
$$

where $g$ is the standard scalar product on $\mathbb{R}^{4 n}=\mathbb{H}^{n}$. The projection $\pi$ is given by the formula

$$
\pi(A)=\frac{1}{2 n}\left(\phi_{I_{0}}(A) I_{0}+\phi_{J_{0}}(A) J_{0}+\phi_{K_{0}}(A) K_{0}\right) .
$$

We shall use the following

Definition 1. Two vectors $X, Y \in \mathbb{H}^{n}$ are called $\mathbb{H}$-dependent if

$$
Y \in \operatorname{span}_{\mathbb{R}}\left\{X, I_{0} X, J_{0} X, K_{0} X\right\} .
$$

Note that if $Y \perp \operatorname{span}_{\mathbb{R}}\left\{X, I_{0} X, J_{0} X, K_{0} X\right\}$ then

$$
\pi(X \wedge Y)=0
$$

and

$$
\|\pi(X \wedge Y)\|^{2}=\frac{1}{2 n}\|X\|^{2}\|Y\|^{2}
$$

if $X, Y$ are $\mathbb{H}$-dependent and $X \perp Y$.

The bundle $\mathcal{G}$ determines a reduction of the $\mathrm{SO}(4 n)$-principal fibre bundle $P$ to the $\operatorname{Sp}(n) \operatorname{Sp}(1)$-principal fibre bundle $Q(M)$ of orthonormal frames $u$ compatible with the almost quaternionic structure given by $\mathcal{G}$, i.e. such that $u I_{0} u^{-1} \in \mathcal{G}, u J_{0} u^{-1} \in \mathcal{G}, u K_{0} u^{-1} \in \mathcal{G}$ where we identify $\mathbb{R}^{4 n}$ with $\mathbb{H}^{n}$ in the standard way. The bundle $\mathcal{G}$ has a natural scalar product defined by $\langle I, J\rangle=-\frac{1}{2} \operatorname{tr} I \circ J$.

Let $u \in Q$. By $\alpha_{u}$ we mean an isomorphism

$$
\alpha_{u}: \mathfrak{s o}(4 n) \rightarrow \bigwedge^{2} T_{p(u)} M
$$


defined by

$$
\alpha_{u}(A)=\sum_{i<j} A_{j}^{i} e_{i} \wedge e_{j}
$$

where $u=\left(e_{1}, \ldots, e_{4 n}\right)$. The mapping $\alpha_{u}$ is an isometry. For fixed $u \in Q$ we can define the projection $\pi_{u}: \bigwedge^{2} T_{p(u)} M \rightarrow \widetilde{\mathcal{G}}=\alpha_{u}(\mathfrak{s p}(1))$ by $\pi_{u}(\gamma)=$ $\alpha_{u}\left(\pi\left(\alpha_{u}^{-1}(\gamma)\right)\right.$. Note that $\pi=\pi_{u}$ is just the orthogonal projection on $\widetilde{\mathcal{G}}$ and does not depend on $u$ and that $\widetilde{\mathcal{G}}$ corresponds (under the isomorphism given by the metric $g$ ) to the bundle $\mathcal{G}$. If $\gamma \in \bigwedge^{2} T_{x} M$ then we write $\gamma^{-}=\gamma_{-}=\pi(\gamma)$. Note that if $\gamma=\alpha_{u}(X)$ where $X \in \mathfrak{g}=\mathfrak{s p}(n) \oplus \mathfrak{s p}(1)$, then $\gamma^{-}=\alpha_{u}\left(X^{-}\right)$. Two vectors $X, Y \in T_{x} M$ are called $\mathbb{H}$-dependent if $Y \in \operatorname{span}\{X, I X, J X, K X\}$ where a quaternionic structure $\{I, J, K\}$ is some local basis of $\mathcal{G}$. The relationships analogous to (1.3a) and (1.3b) hold for any two vectors $X, Y \in T M$.

We let $P^{-}$be the principal $\mathrm{SO}(3)$-bundle of oriented orthogonal bases $\{I, J, K\} \subset \mathcal{G}_{x}, x \in M$ of $\mathcal{G}(I \circ J=K=-J \circ I)$. We have a homomorphism $F: Q \rightarrow P^{-}$of principal fibre bundles defined by

$$
F(u)=\left(u I_{0} u^{-1}, u J_{0} u^{-1}, u K_{0} u^{-1}\right),
$$

which is equivariant with respect to the homomorphism

$$
\Phi: \operatorname{Sp}(n) \mathrm{Sp}(1) \rightarrow \mathrm{SO}(3)
$$

defined by $\Phi(A, q)=\phi_{q} \in \mathrm{SO}(3) \subset \mathrm{Gl}(\operatorname{Im} \mathbb{H})$ where $\phi_{q}(I)=R_{q^{-1}} \circ I \circ R_{q}$ and $R_{q}$ is right multiplication by $q\left(R_{q}(x)=x q\right.$ for $\left.x \in V\right)$ for $I \in \operatorname{Im} \mathbb{H}=$ $\mathbb{R}\left\{I_{0}, J_{0}, K_{0}\right\}$. We denote by $\phi$ the related homomorphism of Lie algebras

$$
\phi: \mathfrak{g}=\mathfrak{s p}(n) \oplus \mathfrak{s p}(1) \rightarrow \mathfrak{s o}(3) .
$$

Note that $\phi=\phi_{+}+\phi_{-}$where $\phi_{+}=\left.\phi\right|_{\mathfrak{s p}(n)}=0$ and $\phi_{-}=\left.\phi\right|_{\mathfrak{s p}(1)}: \mathfrak{g} \rightarrow \mathfrak{s o}(3)$. Consider the mappings of fibre bundles

$$
\Phi_{i}: P^{-}(M) \rightarrow Z(M)
$$

given by $\Phi_{i}\left(J_{1}, J_{2}, J_{3}\right)=J_{i}$. Note that $Z(M)=P^{-}(M) / \mathrm{SO}(2)_{i}=P^{-}(M)$ $\times_{\mathrm{SO}(3)}(\mathrm{SO}(3) / \mathrm{SO}(2))$ where $\mathrm{SO}(2)_{i}=\left\{\phi_{q} \in \mathrm{SO}(\operatorname{Im} \mathbb{H}): \phi_{q} J_{i}=J_{i}\right\}$ for $i \in\{1,2,3\}$ where $J_{1}=I_{0}, J_{2}=J_{0}, J_{3}=K_{0}$, and that $P^{-}(M)$ is a principal $S^{1}$-bundle over $Z(M)$.

Let $(P, \bar{g}),(M, g)$ be Riemannian spaces. A Riemannian submersion $p$ :

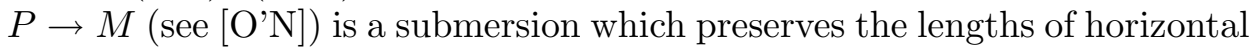
vectors. We shall denote by $V$ the distribution of vertical vectors $(X \in V$ if $X$ is tangent to the fibre $p^{-1}(x)$ for some $\left.x \in M\right)$ and by $H$ the horizontal distribution which is an orthogonal complement of $V$. Let $\mathcal{H}$ and $\mathcal{V}$ denote the projections of the tangent space $T M$ of $M$ onto the subspaces $H, V$, respectively. We denote by $T$ and $A$ the O'Neill tensors defined as follows 
$\left(\right.$ see $\left.\left[\mathrm{O}^{\prime} \mathrm{N}\right]\right)$ :

$$
\begin{aligned}
& T_{X} Y=\mathcal{H}\left(\nabla_{\mathcal{V} X} \mathcal{V} Y\right)+\mathcal{V}\left(\nabla_{\mathcal{V} X} \mathcal{H} Y\right) \\
& A_{X} Y=\mathcal{V}\left(\nabla_{\mathcal{H} X} \mathcal{H} Y\right)+\mathcal{H}\left(\nabla_{\mathcal{H} X} \mathcal{V} Y\right)
\end{aligned}
$$

Finally, recall that a Riemannian manifold $(M, g)$ is called an $\mathcal{A}$-manifold (see [G]) if its Ricci tensor $\varrho$ satisfies the condition $\nabla_{X} \varrho(X, X)=0$ for all $X \in T M$. An $\mathcal{A}$-manifold $M$ is called proper if $\nabla \varrho \neq 0$ and $M$ is locally irreducible. An almost Hermitian manifold $(M, g, J)$ is called an almost Kähler manifold if its Kähler form $\Omega(\Omega(X, Y)=g(X, J Y))$ is closed $(d \Omega=0)$. An almost Kähler manifold is called an almost Hodge manifold if its Kähler form defines a cohomology class which is a (real) multiple of an integral class $([\mathrm{J}-2])$.

2. Killing tensors with two constant eigenvalues. By a Killing tensor on a Riemannian space $(M, g)$ with Levi-Civita connection $\nabla$ we mean a $(1,1)$ tensor $S$ defined on $M$ which satisfies the following conditions:

(a) $g(S X, Y)=g(X, S Y)$ for every $X, Y \in \mathfrak{X}(M)$,

(b) $g(\nabla S(X, X), X)=0$ for every $X \in \mathfrak{X}(M)$.

Definition 2. A distribution $D \subset T M$ is called geodesic if $\nabla_{X} X \in \Gamma(D)$ for every section $X \in \Gamma(D)$.

Proposition 1. Let $(M, g)$ be a Riemannian manifold and let $T M=$ $D_{1} \oplus D_{2}$ where $D_{1}, D_{2}$ are orthogonal geodesic distributions on $M$. Define the tensor $S$ on $M$ by

$$
S X= \begin{cases}\lambda X & \text { if } X \in D_{1}, \\ \mu X & \text { if } X \in D_{2},\end{cases}
$$

where $\lambda, \mu$ are some real numbers. Then $S$ is a Killing tensor. If $\lambda \neq \mu$ and at least one of the distributions $D_{i}$ is not integrable then $\nabla S \neq 0$.

Proof. It suffices to show (see [J-1]) that $\nabla S(X, X)=0$ if $X \in \Gamma\left(D_{i}\right)$ where $i \in\{1,2\}$. Assume for example that $X \in \Gamma\left(D_{1}\right)$. Then from (2.1) we obtain

$$
\nabla S(X, X)=-(S-\lambda \operatorname{Id})\left(\nabla_{X} X\right)=0
$$

since $D_{1}$ is geodesic. It is known that $\nabla S=0$ if and only if both distributions are integrable, which finishes the proof.

Corollary 1. Assume that $p: P \rightarrow M$ is a Riemannian submersion with totally geodesic fibres. Denote by $V, H$ the distributions of vertical and horizontal vectors respectively. Then the tensor $S$ defined by

$$
S X= \begin{cases}\lambda X & \text { if } X \in V, \\ \mu X & \text { if } X \in H\end{cases}
$$


is a Killing tensor. If $\lambda \neq \mu$ and the bundle $p: P \rightarrow M$ is not trivial (i.e. the O'Neill tensor $A \neq 0$ ) then $\nabla S \neq 0$.

Proof. Note that if $p: P \rightarrow M$ is a Riemannian submersion then the distribution $H$ is geodesic. Since the fibres $p^{-1}(x)$ are totally geodesic the distribution $V$ is also totally geodesic.

3. The structure of $\mathcal{A}$-manifold on a principal $\mathrm{SO}(3)$-bundle over a quaternionic-Kähler manifold. Let $P=P(M, G)$ be a principal fibre bundle over a Riemannian manifold $(M, g)$ with a compact, connected, semisimple Lie structure group $G$. Let $\mathfrak{g}$ be the Lie algebra of $G$. Suppose that $\omega \in \mathcal{A}^{1}(P) \otimes \mathfrak{g}$ is a connection form on $P$. Define a metric $g_{c}$ on $P$ by

$$
g_{c}=-c^{2} B(\omega(X), \omega(Y))+p^{*} g(X, Y)
$$

where $B(X, Y)=\operatorname{tr}\left(\operatorname{ad}_{X} \circ \operatorname{ad}_{Y}\right)$ is the Killing form of $\mathfrak{g}$. In [J-1] we have proved the following theorem:

Proposition 2. The group $G$ is a group of isometries of $\left(P, g_{c}\right)$. The orbits of the action of $G$ on $P$ are totally geodesic submanifolds of $P$ isometric to the group $G$ with biinvariant metric given by $-c^{2} B$.

Recall that a Riemannian manifold $(M, g)$ with curvature tensor $R$ is said to have a harmonic curvature if $\delta R=0$ (or equivalently if the Ricci endomorphism $S \in \operatorname{End}(T M)$ is a Codazzi tensor, which means that $\nabla S(X, Y)=$ $\nabla S(Y, X)$ for all $X, Y \in \mathfrak{X}(M))$. Let now $Q$ be the principal $\operatorname{Sp}(n) \operatorname{Sp}(1)$ fibre bundle of orthonormal frames over $(M, g)$. Assume that $\omega \in \mathcal{A}^{1}(Q) \otimes$ $(\mathfrak{s p}(n) \oplus \mathfrak{s p}(1))$ is a connection form associated with the Levi-Civita connection $\bar{\nabla}$ of the metric $g$. Define a metric $g_{c}$ on $Q$ by formula (c). We denote by $H=\{X \in T Q: \omega(X)=0\}$ the horizontal and by $V$ the vertical distribution of $P$. Note that every quaternionic-Kähler manifold is Einstein, hence it has harmonic curvature tensor. Thus we have (see [J-1]):

Proposition 3. The Ricci endomorphism of $\left(Q, g_{c}\right)$ leaves invariant the horizontal and vertical distributions of $Q$.

The homomorphism $F: Q \rightarrow P^{-}$given by (1.4) and the Levi-Civita connection form $\omega$ on $Q$ induce a connection form $\omega^{-} \in \mathcal{A}^{1}\left(P^{-}\right) \otimes \mathfrak{s o}(3)$ on the bundle $P^{-}$such that $\phi_{-} \omega=F^{*} \omega^{-}$. Define the metric

$$
g_{c *}(X, Y)=-\frac{1}{2} c^{2} \operatorname{tr}\left(\omega^{-}(X) \circ \omega^{-}(Y)\right)+p^{*} g(X, Y)
$$

on $P^{-}$. Then (since $(M, g)$ has harmonic curvature tensor and $F^{*} \Omega^{-}=\phi_{-} \Omega$ where $\Omega=D \omega, \Omega^{-}=D \omega^{-}$are the curvature forms of the connections $\Gamma$, $\Gamma^{-}$on $Q, P^{-}$respectively) we have exactly as in [J-1]:

Proposition 4. The Ricci endomorphism of $\left(P^{-}, g_{c *}\right)$ leaves invariant the horizontal and vertical distributions of $\left(P^{-}, g_{c *}\right)$. 
Now we are in a position to prove:

Proposition 5. Let a Riemannian manifold $(M, g), \operatorname{dim} M=4 n$, be a quaternionic-Kähler space with non-zero scalar curvature $\tau_{*}$. Then the principal fibre bundle $p: P^{-} \rightarrow M$ of oriented normed orthogonal bases of the vector bundle $\mathcal{G}$ admits a one-parameter family $P_{c}^{-}$of proper $\mathcal{A}$-structures on $P^{-}$such that $p: P_{c}^{-} \rightarrow M$ is a Riemannian submersion with fibres homothetic to $\mathbb{R P}^{3}=\mathrm{SO}(3)$ with the standard metric of constant curvature.

Proof. Let $F: Q \rightarrow P^{-}$be the homomorphism of principal fibre bundles defined by (1.4). Define a metric $g_{c *}$ on $P^{-}$by $\left(c^{*}\right)$. Below we write $g_{c}$ instead of $g_{c *}$. The natural projection $p: P^{-} \rightarrow M$ is a Riemannian submersion of $P_{c}^{-}=\left(P^{-}, g_{c}\right)$ onto $(M, g)$. From Proposition 2 it follows that O'Neill's tensor $T=0$ (the fibres of $p$ are totally geodesic and homothetic to $\mathrm{SO}(3)=\mathbb{R} P^{3}$ with constant sectional curvature $\left.K=1 /\left(2 n c^{2}\right)\right)$. To compute the tensor $A$ recall that if $X, Y \in T_{F(u)} P^{-}$are horizontal vector fields then

$$
\omega^{-}([X, Y])_{F u}=-\Omega^{-}(X, Y)_{F(u)}=-\phi_{-} \Omega(\bar{X}, \bar{Y})_{u}=-\left(\Omega(\bar{X}, \bar{Y})_{u}\right)^{-}
$$

where $\bar{X}, \bar{Y}$ are horizontal vector fields on $Q$ such that $d F(\bar{X})=X \circ F$. Hence $A_{X} Y$ is a vertical vector field such that $\omega^{-}\left(A_{X} Y\right)=-\frac{1}{2} \Omega^{-}(X, Y)$. If $V$ is a vertical vector field and $d F(\bar{V})=V \circ F$ then

$$
\begin{aligned}
g_{c}\left(A_{X} V, Y\right)_{F(u)} & =-g_{c}\left(V, A_{X} Y\right)_{F(u)}=-\frac{1}{2} c^{2} \operatorname{tr}\left(\omega^{-}(V)_{F(u)} \circ \omega^{-}\left(A_{X} Y\right)_{F(u)}\right) \\
& =\frac{1}{4} c^{2} \operatorname{tr}\left(\omega^{-}(V)_{F(u)} \circ \Omega^{-}(X, Y)_{F(u)}\right) \\
& =\frac{1}{4} c^{2} \operatorname{tr}\left(\phi_{-} \omega(\bar{V})_{u} \circ \phi_{-} \Omega(\bar{X}, \bar{Y})_{u}\right) .
\end{aligned}
$$

We can assume that $X, Y$ are horizontal lifts of vector fields $X_{*}, Y_{*} \in \mathfrak{X}(M)$. Consequently, we obtain

$$
\begin{aligned}
g_{c}\left(A_{X} V, Y\right)_{F(u)} & =\frac{1}{2} c^{2} R\left(X_{*} \wedge Y_{*}, \alpha_{u}\left(\omega^{-}(V)\right)\right) \\
& =\frac{1}{2} c^{2} g\left(X_{*} \wedge Y_{*}, R_{\mid \mathcal{G}}\left(\alpha_{u} \omega^{-}(V)\right)\right)
\end{aligned}
$$

and thus, in view of $(\mathrm{R})$,

$$
g_{c}\left(A_{X} V, Y\right)_{F(u)}=\frac{1}{2} c^{2} \alpha g\left(X_{*} \wedge Y_{*}, \alpha_{u}\left(\omega^{-}(V)\right)\right) .
$$

Let $\left\{V_{1}, V_{2}, V_{3}\right\}$ be an orthonormal basis of $\mathfrak{s p}(1)$ and let $\left\{v_{1}, v_{2}, v_{3}\right\}$ be the Killing vector fields corresponding (under the homomorphism $F$ ) to $V_{i}$. Then $g_{c}\left(v_{i}, v_{i}\right)=c^{2}$ and $g\left(\alpha_{u}\left(V_{i}\right), \alpha_{u}\left(V_{i}\right)\right)=1$. Hence

$$
c^{2}\left\|A_{X} Y\right\|^{2}=\sum_{i=1}^{3} g_{c}\left(A_{X} Y, v_{i}\right)^{2}=\frac{1}{4} c^{4} \sum_{i=1}^{3} g\left(X_{*} \wedge Y_{*}, R_{\mid \mathcal{G}}\left(\alpha_{u}\left(V_{i}\right)\right)\right)^{2} .
$$


From (3.3) it follows that if $\|X\|=\|Y\|=1, X, Y$ are $\mathbb{H}$-dependent and $X \perp Y$ then (see (1.3a) and (1.3b))

$$
\left\|A_{X} Y\right\|^{2}=\frac{1}{4} c^{2} \alpha^{2}\left\|\left(X_{*} \wedge Y_{*}\right)_{-}\right\|^{2}=\frac{1}{8 n} c^{2} \alpha^{2}
$$

and $\left\|A_{X} Y\right\|=0$ if $X \perp \mathcal{G} Y=\mathbb{R}\{I Y, J Y, K Y\}$. Let $\left\{Y_{1 *}, \ldots, Y_{4 n *}\right\}$ be a local orthonormal frame on $M$ such that any four vectors $Y_{4 k+1}, Y_{4 k+2}, Y_{4 k+3}$, $Y_{4 k+4}$ are $\mathbb{H}$-dependent for $k=0,1, \ldots, n-1$. Let $\left\{Y_{1}, \ldots, Y_{4 n}\right\}$ be its horizontal lift. We can assume that $X=Y_{1}$. Note that if $X$ is horizontal and $\|X\|=1$ then for a vertical vector field $V$ we have

$$
\begin{aligned}
\left\|A_{X} V\right\|^{2} & =\sum_{i=1}^{4} g_{c}\left(A_{X} V, Y_{i}\right)^{2} \\
& =\frac{1}{4} c^{4} \sum_{i=1}^{4} g\left(\left(X_{*} \wedge Y_{i_{*}}\right)_{-}, R_{\mid \mathcal{G}}\left(\alpha_{u}\left(\omega^{-}(V)\right)\right)\right)^{2} \\
& =\frac{1}{8 n} c^{4} \| R_{\mid \mathcal{G}}\left(\alpha_{u}\left(\omega^{-}(V)\right) \|^{2}\right. \\
& =\frac{1}{8 n} c^{4} \alpha^{2}\left\|\alpha_{u}\left(\omega^{-}(V)\right)\right\|^{2}=\frac{1}{8 n} c^{2} \alpha^{2}\|V\|^{2}
\end{aligned}
$$

in view of the relation $c^{2}\left\|\alpha_{u}(\omega(V))\right\|^{2}=g_{c}(V, V)$. Hence we have (we assume that $\|X\|=\|Y\|=1, X \perp Y$ and $P_{X Y}$ denotes the plane spanned by $\{X, Y\}$ )

$$
\begin{array}{ll}
K\left(P_{v_{i} v_{j}}\right)=\frac{1}{2 n c^{2}} \quad \text { if } i \neq j, & K\left(P_{X v_{i}}\right)=\frac{1}{8 n} c^{2} \alpha^{2}, \\
K\left(P_{X Y}\right)=K\left(P_{X_{*} Y_{*}}\right)-\frac{3}{8 n} c^{2} \alpha^{2} & \text { if } X, Y \text { are } \mathbb{H} \text {-dependent, } \\
K\left(P_{X Y}\right)=K\left(P_{X_{*} Y_{*}}\right) & \text { if } X \perp \operatorname{span}\{I Y, J Y, K Y\}=\mathcal{G} Y .
\end{array}
$$

Consequently, if $V$ is a vertical vector field such that $\|V\|=1$ then

$$
\varrho(V, V)=\frac{1}{n c^{2}}+\frac{1}{2} c^{2} \alpha^{2}
$$

and if $X$ is horizontal and $\|X\|=1$ then

$$
\varrho(X, X)=\frac{1}{4 n} \tau_{*}-3 \frac{3}{8 n} c^{2} \alpha^{2}+\frac{3}{8 n} c^{2} \alpha^{2}=\frac{(n+2) \alpha}{n}-\frac{3}{4 n} c^{2} \alpha^{2} .
$$

From Proposition 4 we get

$$
\varrho(\xi, X)=0
$$

for any vertical vector field $\xi$ and any horizontal vector field $X$, i.e. the Ricci endomorphism $S$ of $\left(P_{c}^{-}, g_{c}\right)$ preserves the horizontal and vertical distributions. From (3.5) and (3.6) we deduce that $S$ has exactly two constant 
eigenvalues

$$
\lambda=\frac{1}{n c^{2}}+\frac{1}{2} c^{2} \alpha^{2} \text { and } \mu=\frac{(n+2) \alpha}{n}-\frac{3}{4 n} c^{2} \alpha^{2} .
$$

Note that $\lambda=\mu$ if and only if $c^{2}=2 / \alpha$ or $c^{2}=2 /((2 n+3) \alpha)$ and for those values of $c$ the manifold $P_{c}^{-}$is an Einstein space. Denote by $H$ the horizontal distribution of the submersion $p_{c}: P_{c}^{-} \rightarrow M$. Since for $\lambda \neq \mu$ the distribution $D_{0}=\operatorname{span}\left\{v_{1}, v_{2}, v_{3}\right\}$ is an eigendistribution of the Ricci tensor which is totally geodesic and the leaves of the foliation $D_{0}$ are locally irreducible it follows that if $P_{c}^{-}$were locally reducible we would have $D_{0}^{\perp}=H=D_{1} \oplus D_{2}$ where $D_{0} \oplus D_{1}$ and $D_{2}$ would be parallel distributions. For a Killing vector field $\xi=v_{1} \in D_{0}$ define $T=\nabla \xi$. Then $T$ is skew-symmetric, $T D_{0} \subset D_{0}$ and $T D_{2} \subset D_{1}, T D_{1} \subset D_{1}$. Thus we would have $T X=0$ for every $X \in D_{2}$, contrary to (3.4). It follows that $P_{c}^{-}$is locally irreducible. For $c^{2} \neq 2 / \alpha$ and $c^{2} \neq 2 /((2 n+3) \alpha)$ from Proposition 1 it follows that $\left(P^{-}, g_{c}\right)$ is a proper $\mathcal{A}$-manifold.

Hence we get

COROLlary 2. Let $(M, g)$ be a quaternionic-Kähler manifold with nonzero scalar curvature $\tau_{*}$. For $c^{2}=2 / \alpha$ or $c^{2}=2 /((2 n+3) \alpha)$ where $\alpha=$ $\tau_{*} /(4(n+2))$ the manifold $P_{c}^{-}$is an Einstein space. For other $c^{2}$ the manifold $\left(P_{c}^{-}, g_{c}\right)$ is a proper $\mathcal{A}$-manifold whose Ricci tensor has exactly two constant eigenvalues

$$
\lambda=\frac{1}{n c^{2}}+\frac{1}{2} c^{2} \alpha^{2} \quad \text { and } \quad \mu=\frac{(n+2) \alpha}{n}-\frac{3}{4 n} c^{2} \alpha^{2} .
$$

If $c^{2}<4(n+2) /(3 \alpha)$ then $\left(P_{c}^{-}, g_{c}\right)$ has positive Ricci curvature.

4. Almost Kähler $\mathcal{A}$-structure on the twistor bundle $Z(M)$. Let $(P, g)$ be a Riemannian manifold and $\xi$ be a unit Killing vector field on $P$. Define a tensor field $\phi$ by $\phi(X)=\nabla_{X} \xi$ and a 1 -form $\eta$ by $\eta(X):=g(\xi, X)$. Then we call $(P, g, \xi, \phi, \eta)$ a $K$-contact structure if the following relation is satisfied:

$$
\phi^{2}=-\mathrm{id}+\eta \otimes \xi
$$

Assume that $(P, g)$ is a circle bundle over a manifold $M$ and that the fundamental vector field of the action of the group $S^{1}$ is a constant multiple of a unit Killing vector $\xi$ on $P$. If $\xi$ gives a $\mathrm{K}$-contact structure $(\xi, \phi, \eta)$ on $P$ then there is a unique metric $g_{*}$ and a unique almost complex structure $J_{*}$ on $M$ such that $\left(M, g_{*}, J_{*}\right)$ is an almost Kähler manifold and

$$
d \eta(X, Y)=g(X, \phi Y)=p^{*} \Omega(X, Y)
$$

where $\Omega$ is the Kähler form of $\left(M, g_{*}, J_{*}\right)$ (i.e. $\Omega(X, Y)=g\left(X, J_{*} Y\right)$ ). Assume that $c^{2}=1 /(2 n)$ (see $\left.\left(\mathrm{c}^{*}\right)\right)$ and let $g=g_{1 /(2 n)}$ be the fixed metric 
on $P^{-}$. Hence

$$
g(X, Y)=-\frac{1}{4 n} \operatorname{tr}\left(\omega^{-}(X) \circ \omega^{-}(Y)\right)+p^{*} g_{0}(X, Y)
$$

where $g_{0}$ is the metric on the quaternionic-Kähler manifold $M$. Then the vector fields $\left(V_{1}, V_{2}, V_{3}\right)$ corresponding via $F$ to $v_{1}=I_{0}, v_{2}=J_{0}, v_{3}=K_{0} \in$ $\mathfrak{s p}(1) \subset \mathfrak{s o}(4 n)$ are unit Killing vector fields (in view of Proposition 2). We start with:

Proposition 6. Assume that $\left(M, g_{0}\right)$ is a quaternionic-Kähler manifold with scalar curvature $\tau_{0}$ such that $\left|\tau_{0}\right|=16 n(n+2)$. Then the fields $V_{1}, V_{2}, V_{3}$ define on $\left(P^{-}, g\right)$ three $K$-contact structures. The tensors $\phi_{i}$ induce on $\left(Z(M), g_{*}\right)$ the almost Kähler structure $J_{*}$ which does not depend on $i$.

Proof. It is clear that $V_{i}$ is a unit Killing field. Define the 1-form $\eta_{i}$ by $\eta_{i}(X)=g\left(V_{i}, X\right)$. We only have to show that $\phi_{i}=\nabla V_{i}$ defines on $H_{i}=\operatorname{ker} \eta_{i}$ an almost complex structure. Note that $\phi_{i}(X)=A_{X} V_{i}$ if $X$ is horizontal and that $\phi_{i}$ preserves the horizontal and vertical bundles. From (3.2) it follows that the restriction of $\phi_{i}$ to the horizontal distribution is an almost complex structure. In fact we have $\alpha=4 n \varepsilon$ and

$$
g\left(A_{X} V_{i}, Y\right)=\frac{1}{2} c^{2} \alpha g\left(X_{*} \wedge Y_{*}, \alpha_{u}\left(v_{i}\right)\right)=\varepsilon g\left(X_{*}, J_{\alpha_{u}\left(v_{i}\right)} Y_{*}\right)
$$

where $X, Y$ are horizontal with respect to the submersion $p: P^{-} \rightarrow M, \varepsilon=$ $\operatorname{sgn} \tau_{0}$ and $p(X)=X_{*}, p(Y)=Y_{*}$. On the other hand the restriction to the vertical distribution also gives an almost complex structure (on the fibres, $\Phi_{i}$ (see (1.5)) is just the Hopf fibration $\mathbb{R} P^{3} \rightarrow \mathbb{C} P^{1}$ ). The last statement of the proposition follows from the fact that $J_{*}$ is one of the two natural almost complex structures $J_{+}, J_{-}$on $Z(M)$ whose definitions we recall below. The theorem follows from the well known properties of K-contact structures (see $[\mathrm{J}-2],[\mathrm{J}-3])$.

Below the following definition will be useful:

Definition 3. Assume that a Lie group $G(G=\mathrm{SO}(3)$ or $G=\mathrm{SU}(2))$ acts by isometries on a Riemannian manifold $(M, g)$ in such a way that the orbits $\operatorname{orb}_{G}(x)$ of the action are totally geodesic submanifolds isometric to $G$ with its canonical metric of constant sectional curvature $K=1$. Let $\left\{\xi_{1}, \xi_{2}, \xi_{3}\right\}$ be Killing vector fields corresponding to the generators of the Lie algebra $\mathfrak{s o}(3)$ and denote by $H$ the orthogonal complement of $\operatorname{span}\left\{\xi_{1}, \xi_{2}, \xi_{3}\right\}$. Assume also that every vector field $\xi_{i}$ gives a K-contact structure. Hence

$$
\left[\xi_{i}, \xi_{j}\right]=2 \varepsilon_{i j k} \xi_{k} \quad \text { and } \quad \nabla_{\xi_{i}} \xi_{j}=\varepsilon_{i j k} \xi_{k} .
$$

Let the fields $\xi_{1}, \xi_{2}, \xi_{3}$ satisfy the condition (for pairwise different $i, j, k$ )

$$
J_{i} \circ J_{j}=\varepsilon \varepsilon_{i j k} J_{k},
$$


i.e. $\left(\varepsilon J_{1}, \varepsilon J_{2}, \varepsilon J_{3}\right)$ is an almost quaternionic-structure on $H$, where $\phi_{i}=\nabla \xi_{i}$ and $J_{i}=-\phi_{i \mid H}$. Then we call $\left(M, \xi_{i}, \phi_{i}, \eta_{i}\right)$ a 3 -K-contact structure if $\varepsilon=1$ and a quasi 3 -K-contact structure if $\varepsilon=-1$.

REMARK. Note that if $\varepsilon=1$ then the above definition is equivalent to the usual definition of a $3-K$-contact structure (see $[\mathrm{K}],[\mathrm{Ku}]$ and $[\mathrm{J}-3]$ ). The notion of $\mathrm{nS}$-structure introduced by $\mathrm{S}$. Tanno is stronger than the notion of quasi 3-K-contact structure and the relation between these two notions is similar to that between the 3-K-contact structure and 3-Sasakian structure in the positive case. An nS-structure is a quasi 3-K-contact structure satisfying an additional condition similar to the condition of being Sasakian in the positive case.

Proposition 7. Let an oriented Riemannian 4n-manifold $(M, g)$ be a quaternionic-Kähler manifold with scalar curvature $\tau_{0}$ such that $\left|\tau_{0}\right|=$ $16 n(n+2)$. Then the fibre bundle $p: P^{-} \rightarrow M$ furnished with the metric $g$ admits a 3-K-contact structure if $\tau_{0}>0$ and a quasi 3 -K-contact structure if $\tau_{0}<0$.

P r o of. We prove that the triple $\left(V_{1}, V_{2}, V_{3}\right)$ gives the required structure on $P^{-}(M)$. Define $\Psi_{i}=\Phi_{i} \circ F: Q(M) \rightarrow Z(M)$ (see (1.5)). Then $\Psi_{i}(u)=$ $\alpha_{u}\left(v_{i}\right)$. Note that $p:\left(P^{-}(M), g\right) \rightarrow\left(M, g_{0}\right)$ is a Riemannian submersion. Let $u \in Q$ and $v=F(u) \in P^{-}(M)$. Define $H_{v}=\left\{X \in T P^{-}: \eta_{1}(X)=\right.$ $\left.\eta_{2}(X)=\eta_{3}(X)=0\right\}$. Then $p: H_{v} \rightarrow T_{p(v)} M$ is an isometry and $J_{i}=-\phi_{i \mid H_{v}}$ is given by $J_{i}=\varepsilon J_{\Psi_{i}(u)}$, where we identify $H_{v}$ with $T_{p(v)} M$ via $p$ and where $\varepsilon$ is the sign of $\tau_{0}$. Note that $J_{i} \circ J_{j}=\varepsilon \varepsilon_{i j k} J_{k}$ (since $v_{i} v_{j}=\varepsilon_{i j k} v_{k}$ ). Thus the assumptions of Definition 3 are satisfied, which completes the proof.

REMARK. The case $\tau_{0}>0$ of Proposition 7 is well known. Note that if $\tau_{0}>0$ then our 3 -K-contact structure is 3-Sasakian. The case $\tau_{0}<0$ was proved earlier in a different way by S. Tanno (see [T], Th. B). In fact Tanno proved more: he showed that our quasi 3-K-contact structure is in fact an nS-structure. He also described the Ricci tensor of $\left(P^{-}, g\right)$.

Let $\left(M, g_{0}\right)$ be a quaternionic-Kähler manifold. We have two natural almost complex structures $J_{-}, J_{+}$on the twistor bundle $\pi: Z(M) \rightarrow M$. Both structures preserve the vertical and horizontal distributions of $Z(M)$. They are given as follows (see $[\mathrm{E}-\mathrm{S}])$ :

$$
g_{*}\left(X, J_{+} Y\right)=g_{0}\left(\pi(X), J_{\omega} \pi(Y)\right)=g_{*}\left(X, J_{-} Y\right)
$$

if $X, Y$ are horizontal with respect to $\pi: Z(M) \rightarrow M$, hence on $H_{\omega} \approx$ $T_{\pi(\omega)} M$ the almost complex structures $J_{+}=J_{-}$are just $J_{\omega}$. Write $\xi=V_{1}$, $\Phi=\Phi_{1}$. If $X$ is a vertical vector then we define

$$
J_{+} X=-d \Phi\left(\nabla_{X^{*}} \xi\right), \quad J_{-} X=d \Phi\left(\nabla_{X^{*}} \xi\right)
$$


where $X^{*}$ is the horizontal lift of $X \in V Z(M)$ with respect to the Riemannian submersion $\Phi: P^{-} \rightarrow Z$. It is well known (see [B]) that the structure $J_{+}$is integrable, while $J_{-}$is not. In what follows we also write $J_{1}=J_{+}$and $J_{-1}=J_{-}$.

In my paper [J-2] I have proved that a regular K-contact manifold $P$ (i.e. $P$ is a principal $S^{1}$-bundle over $M$ and $p:(P, g) \rightarrow\left(M, g_{*}\right)$ is a Riemannian submersion) is an $\mathcal{A}$-manifold if and only if $\left(M, g_{*}\right)$ with the induced almost Kähler structure $J_{*}$ is an almost Kähler $\mathcal{A}$-manifold whose Ricci tensor $\varrho_{*}$ is Hermitian, i.e. $\varrho_{*}\left(J_{*} X, J_{*} Y\right)=\varrho_{*}(X, Y)$ for all $X, Y \in \mathfrak{X}(M)$.

Proposition 8. Let $\left(M, g_{0}\right)$ be a quaternionic-Kähler manifold with scalar curvature $\tau_{0}=16 n(n+2) \varepsilon$ where $\varepsilon \in\{-1,1\}$. Then the manifold $\left(Z(M), g_{*}, J_{\varepsilon}\right)$ is an almost Kähler $\mathcal{A}$-manifold. If $\tau_{0}>0$ then $\left(Z(M), g_{*}, J_{+}\right)$ is Kähler-Einstein and the 3-K-contact structure on $\left(P^{-}(M), g\right)$ is a 3Sasakian structure. If $\tau_{0}<0$ then $\left(Z(M), g_{*}, J_{-}\right)$is a strictly almost Hodge $\mathcal{A}$-manifold whose Ricci tensor has two constant eigenvalues $\lambda_{*}=4 n+4$ and $\mu_{*}=-4 n-12$ of multiplicity 2 and $4 n$ respectively. The Ricci tensor $\varrho_{*}$ of $\left(Z(M), g_{*}\right)$ is Hermitian with respect to both structures $J_{+}, J_{-}$, i.e. $\varrho_{*}\left(J_{+} X, J_{+} Y\right)=\varrho_{*}\left(J_{-} X, J_{-} Y\right)=\varrho_{*}(X, Y)$. If $M$ is compact then $Z(M)$ is a compact space.

Pr o o f. Note that $\left(P^{-}, g\right)$ is an $\mathcal{A}$-manifold (see [J-1]). Let $\Phi=\Phi_{1}$ and $\xi=V_{1}$. Then the Killing field $\xi$ defines on $\left(P^{-}, g\right)$ a K-contact structure and $\Phi: P^{-} \rightarrow Z(M)$ is an $S^{1}$-principal fibre bundle. Thus from Theorem 1 of [J-2] it follows that $\left(Z(M), g_{*}, J_{*}\right)$ is an almost Kähler $\mathcal{A}$-manifold with Hermitian Ricci tensor. From (4.1)-(4.3) it follows that the almost Kähler structure $J_{*}$ induced on $Z(M)$ by $\phi=\nabla \xi$ coincides with $-J_{+}$if $\tau_{0}>0$ and with $J_{-}$if $\tau_{0}<0$. Hence if $\tau_{0}>0$ then $J_{*}$ is integrable. Consequently, $\left(Z(M), g_{*}, J_{+}\right)$is a Kähler manifold. Thus every Killing vector field $V_{i}$ gives a Sasakian structure on $\left(P^{-}(M), g\right)$ and the triple $\left(V_{1}, V_{2}, V_{3}\right)$ defines a 3 Sasakian structure on $\left(P^{-}(M), g\right)$ (see $\left.[\mathrm{K}]\right)$. It is also an Einstein space since from Corollary 2 it follows that for $c^{2}=1 /(2 n)$ and $\alpha=4 n$ the manifold $\left(P^{-}, g\right)$ is Einstein. Thus $\left(Z(M), g_{*}\right)$ is also Einstein in this case (see also [B] and $[\mathrm{S}])$.

If $\tau_{0}<0$ then the Ricci tensor $S$ of $\left(P^{-}, g_{c}\right)$ has two constant eigenvalues whose eigendistributions are just the vertical and horizontal distributions. If $c^{2}=1 /(2 n)$ and $\alpha=-4 n$ then the eigenvalues are $\lambda=4 n+2$ and $\mu=-4 n-$ 14. Hence it follows from [J-2] that the Ricci tensor $S_{*}$ of $\left(Z(M), g_{*}\right)$ has exactly two eigenvalues $\lambda_{*}=\lambda+2=4 n+4$ and $\mu_{*}=\mu+2=-4 n-12$ whose eigendistributions are the vertical and horizontal distributions respectively. Since $J_{+}, J_{-}$also preserve the vertical and horizontal distributions it follows that $S_{*} \circ J_{\varepsilon}=J_{\varepsilon} \circ S_{*}$, which means that the Ricci tensor of $\left(Z(M), g_{*}\right)$ is Hermitian with respect to both structures. If $\tau_{0}<0$ then $J_{*}=J_{-}$is not 
integrable and from the construction it is clear that $\left(Z(M), g, J_{*}\right)$ is a strictly almost Hodge $\mathcal{A}$-manifold. The scalar curvature $\tau_{*}$ of $\left(Z(M), g_{*}\right)$ is negative, $\tau_{*}=-8 n(2 n+5)+8$.

REMARK. Note that all the above results are proved by different methods in [A-G-I] (with the exception of the fact that $Z(M)$ is an almost Hodge $\mathcal{A}$-manifold). Let us recall the Goldberg conjecture: A compact Einstein almost Kähler manifold is Kähler, formulated by S. I. Goldberg [Go] almost thirty years ago. This conjecture was proved by K. Sekigawa [Se] under the assumption that the scalar curvature is non-negative. The case of negative scalar curvature is still open. In relation to this conjecture, examples of strictly almost Kähler Riemannian manifolds which are close to Einstein are of great interest (see $[\mathrm{D}-\mathrm{M}]$ ). Compact examples are of particular interest. Note that there are examples of compact quaternionic-Kähler manifolds in any dimension $4 n$ (for example compact quotients of symmetric spaces dual to the symmetric spaces discussed by Wolf [W]). In that way we obtain examples of compact almost Hodge $\mathcal{A}$-manifolds with Hermitian Ricci tensor in every dimension $4 n+2$ (see also [J-2]). These examples indicate that the Goldberg conjecture may be false in general.

Acknowledgments. I am very grateful to P. Kobak for many discussions on twistor bundles and to K. Galicki whose lectures at the Banach Center in Warsaw and in Cracow introduced me to 3-Sasakian structures. The author is also grateful to the referee for his useful remarks.

\section{References}

[A-G-I] B. Alexandrov, G. Grantcharov and S. Ivanov, Curvature properties of twistor spaces of quaternionic-Kähler manifolds, J. Geometry 62 (1998), 1-12.

[B] A. Besse, Einstein Manifolds, Springer, Berlin, 1987.

[D-N] J. E. D'Atri and H. K. Nickerson, Divergence-preserving geodesic symmetries, J. Differential Geom. 3 (1969), 467-476.

[D-M] J. Davidov and O. Muskarov, Twistor spaces with Hermitian Ricci tensor, Proc. Amer. Math. Soc. 109 (1990), 1115-1120.

[E-S] J. Eells et S. Salamon, Constructions twistorielles des applications harmoniques, C. R. Acad. Sci. Paris 296 (1983), 685-687.

[G-L] K. Galicki and H. B. Lawson, Quaternionic reduction and quaternionic orbifolds, Math. Ann. 282 (1988), 1-21.

[Go] S. I. Goldberg, The integrability of almost Kähler structures, Proc. Amer. Math. Soc. 21 (1969), 96-100.

[G] A. Gray, Einstein like manifolds which are not Einstein, Geom. Dedicata 7 (1978), 259-280.

[J-1] W. Jelonek, Self-duality and $\mathcal{A}$-manifolds, J. London Math. Soc. 58 (1998), 697-708.

[J-2] -, K-contact $\mathcal{A}$-manifolds, Colloq. Math. 75 (1998), 97-103. 
[J-3] W. Jelonek, Almost Kähler A-structures on twistor bundles, Ann. Global Anal. Geom. 17 (1999), 329-339.

[K] M. Konishi, On manifolds with Sasakian 3-structures over quaternionic-Kähler manifolds, Kodai Math. Sem. Rep. 26 (1975), 194-200.

[Ku] Y.-Y. Kuo, On almost contact 3-structures, Tôhoku Math. J. 22 (1970), 325332.

[O'N] B. O'Neill, The fundamental equations of a submersion, Michigan Math. J. 13 (1966), 459-469.

[S] S. Salamon, Quaternionic Kähler manifolds, Invent. Math. 67 (1982), 143171.

[Se] K. Sekigawa, On some compact Einstein almost Kähler manifolds, J. Math. Soc. Japan 36 (1987), 101-116.

[Sw] A. Swann, Hyperkähler and quaternionic Kähler geometry, Math. Ann. 289 (1991), 421-450.

[T] S. Tanno, Remarks on a triple of K-contact structures, Tôhoku Math. J. 48 (1996), 519-531.

[W] J. A. Wolf, Complex homogeneous contact manifolds and quaternionic symmetric spaces, J. Math. Mech. 14 (1965), 1033-1047.

Institute of Mathematics

Cracow University of Technology

Warszawska 24

31-155 Kraków, Poland

E-mail:wjelon@usk.pk.edu.pl

Reçu par la Rédaction le 1.10.1999

Révisé le 15.2.2000 